\title{
Formulation and Evaluation of Meloxicam In-situ Gel Designed for Sustained Drug Release to Reduce Dosing Frequency in the Management of Arthritis
}

\author{
Dr. Srinivasa Rao Meesala*, Iswariya V T, Dr. M. S Chandra, Dr. C. Venkataramana Reddy \\ Assistant Dean School of agriculture science \& Department of Pharmaceutical Biotechnology, \\ Department of Agriculture and Pharmaceutical Biotechnology, K.K. University, Nepura, Bihar Sharif, Nalanda, Bihar, India. \\ *Corresponding author's E-mail: nivasbiotech1979@gmail.com
}

Received: 14-05-2021; Revised: 18-07-2021; Accepted: 26-07-2021; Published on: 15-08-2021.

\begin{abstract}
Meloxicam has short biological half-life and is rapidly eliminated, frequent oral administration is necessary to maintain its therapeutic concentration, but this can increase chances of missing dose. This makes Meloxicam a good applicant for oral sustained release formulation. The objective of study was to develop in-situ gel formulations of Meloxicam for sustained release to reduce the dosing frequency in the treatment of rheumatoid arthritis. Method of Ion sensitive in-situ gelation was used in this study. Meloxicam In-situ gel formulations were prepared by varying concentrations of sodium alginate as a bio-degradable gel forming polymer, $\mathrm{CaCl} 2$ as a cross-linking agent and Chitosan/ HPMCK4/HPMCK15/Guar gum/Gellan gum/ Xantha gum/pectin were used as drug release rate controlling polymers. The formulations F11-F18 were assessed for Physical appearance, $\mathrm{pH}$, in-vitro drug release, viscosity, in-vitro gelling capacity and drug content. FTIR, DSC and in-vivo drug kinetics studies was conducted for Meloxicam, excipients used and optimized formulation. Formulations showed an optimum viscosity that will allow ease of administration and swallowing. All formulations are shown $\mathrm{pH}$ between4.7-4.9, floating lag time was $2-3 \mathrm{sec}$ and floated for $>12 \mathrm{hrs}$. In vitro drug release studies reporting that commercially available product Meloxicam SR has showed $99.92 \%$ drug release in 8 hrs and out of eight formulations F11 showing in-vitro drug release of $99.52 \%$ over a $12 \mathrm{hrs}$ extended period. FTIR studies revealed no interaction between drug and excipients used. The results of In-vivo kinetic studies are approving the better performance of the optimized formulation in comparison to marketed formulation, The Cmax, Tmax, half-life AUC values are confirming the same thing. In conclusion, Formulation (F11) was selected as optimized formulations could be offered as shows optimum sustained drug release compared to commercial formulation. Hence Meloxicam containing Chitosan as drug release controlling polymer as promising approach for the treatment of rheumatoid arthritis treatment in a convenient dosage form with much patient compliance and better therapeutic response.
\end{abstract}

Keywords: Meloxicam, Arthritis, In-situ gels, sodium alginate, Chitosan.

QUICK RESPONSE CODE $\rightarrow$

DOI:

10.47583/ijpsrr.2021.v69i02.025

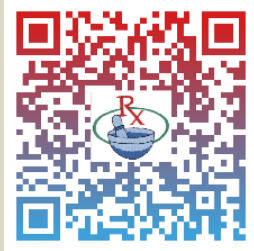

DOI link: http://dx.doi.org/10.47583/ijpsrr.2021.v69i02.025

\section{INTRODUCTION}

A

rthritis is an "autoimmune disease described by joint inflammation and pain/ache, with progressive bone erosions and cartilage damage, accompanied as a result of synovial hypertrophy"1,2. The causing reason of disease is unidentified and above $1 \%$ of world population suffering from Rheumatoid arthritis, characteristically Arthritis disease commencement at the age of 30 to 50 years ${ }^{3}$. An expected 2.5 million individuals in India is pretentious by Rheumatoid arthritis and has economic impact in billions. About $0.5-0.75 \%$ of Asian people has been suffering from Arthritis with a woman to man ratio $3: 1^{4}$. In addition RA promotes rate of premature death, patients with savage ischemic coronary illness or lymphoma which extraordinarily effects on monetary $\operatorname{cost}^{5,6}$.
In spite of progressions in fundamental science and therapeutics, the present available treatments are partially effective because of several disadvantages like drug dosage, time of administration and related toxicities ${ }^{7}$. The perfect system should be easily administered, sustained drug release manner and optimum concentration of drug must be retaining without unpleasant negative activities. A variety of therapeutic agents are obtainable for the management of Arthritis consists of (i) NSAIDs (nonsteroidal anti-inflammatory drugs)/selective cyclo oxygenase-2 (COX-2) inhibitors, (ii) Disease-modifying antirheumatic drugs (DMARDs), (iii) Glucocorticoids, (iv) Natural origin compounds and (v) Biological agents ${ }^{8}$.

Meloxicam is an exceptionally strong anti-inflammatory non-steroidal drug, it has been utilized efficaciously in the therapeutic management of an extensive range of inflammatory and painful conditions, for example, moderate to chronic rheumatoid joint inflammation (RA), ankylosing-spondylitis and osteoarthritis ${ }^{9}$.

The main aim of study was formulation and evaluation of Meloxicam in-situ gel preparation for sustained drug release to reduce dosing frequency in a convenient dosage form for geriatric patients in the treatment of rheumatoid arthritis. 


\section{MATERIALS AND METHODS}

Meloxicam was acquired from Glenmark Pharma private Ltd, Mumbai, India. All the polymers received were of pharmaceutical grade (Chitosan/ HPMCK4/HPMCK15 are obtained from Lepid Life Sciences Pvt Ltd, Delhi, India and Guar gum/Gellan gum/ Xantha gum/pectins are obtained from Sigma-Aldrich Germany) were used as received. Sodium alginate, Sodium citrate and Calcium chloride were obtained from S.D Fine chemicals, Mumbai, India. All chemicals and solvents utilized were of HPLC grade. Throughout the study distilled water was used.

\section{Determination of absorption maximum $\left(\lambda_{\max }\right)$ of Meloxicam ${ }^{10}$ and construction of calibration curve}

The standard solution of drug was prepared in methanol and examined at 200 to $400 \mathrm{~nm}$ range using Elico UV spectrophotometer, to find out absorption maxima for analysis of samples. Meloxicam $10 \mathrm{mg}$ of pure drug was dissolved in methanol and made upto $10 \mathrm{ml}$ with $0.1 \mathrm{~N} \mathrm{HCl}$ by slight shaking $(1000 \mathrm{mcg} / \mathrm{ml})$ and second stock solution was prepared by diluting $1 \mathrm{ml}$ of standard to $10 \mathrm{ml}$ with addition of $0.1 \mathrm{~N} \mathrm{HCl}$, which gives $100 \mathrm{mcg} / \mathrm{ml}$. As of concentrations like $10,20,30,40$ and $50 \mu \mathrm{g} / \mathrm{ml}$ in $0.1 \mathrm{~N}$ $\mathrm{HCl}$ were prepared and absorbance's were recorded. A standard graph was plotted and calculated the correlation coefficient of Meloxicam.

\section{Preparation of Meloxicam in-situ gels formulation}

Ion sensitive in-situ gelation method was employed for preparation of Meloxicam in-situ gels and charts of formulation specified in table no 1 . In preparation of insitu gels sodium alginate used as a Gelling agent, Sodium citrate as a sequestering agent and cross linking agent was Calcium chloride apart from these, polymers like Chitosan/ HPMCK4/HPMCK15/Guar gum/Gellan gum/ Xantha gum/pectins were utilized as rug release rate controlling polymer.

On addition of formulations (F1-F3) to $0.1 \mathrm{~N} \mathrm{HCl}, \mathrm{F} 1$ - sol to gel transformation occurred very slowly. Transparent, less compact and freely pourable gel was formed. In case of F2 - Sol to gel transformation is not very slow and formed transparent and nice matrix. F3 - Sol to gel transformation occurred immediately and formed opaque gel. Therefore Increasing concentration of Sodium Alginate resulted in producing an intact gel which could reside for longer period of time but further rise in concentration leads so further opaque gel which is not acceptable. So $3 \%$ Sodium Alginate is selected for further trials.

To know the effect of calcium chloride concentration, on gelling time and gel consistency, F4 to F7 preparations were designed by increasing concentration of calcium chloride.

There is no-detectable change in the gelling time and gel consistency; hence the least concentration is selected for further formulations preparation. Further trials were planned by adding drug and drug release controlling polymer at the concentration of $0.1 \%$.

\begin{tabular}{|c|c|c|c|c|c|c|c|}
\hline Ingredient & F1 & F2 & F3 & F4 & F5 & F6 & F7 \\
\hline Sodium alginate $(g)$ & 1 & 2 & 3 & 2 & 3 & 2 & 3 \\
\hline Sodium citrate(g) & 0.05 & 0.05 & 0.05 & 0.05 & 0.05 & 0.05 & 0.05 \\
\hline Calciumchloride $(\mathrm{g})$ & 0.05 & 0.05 & 0.05 & 0.1 & 0.1 & 0.15 & 0.15 \\
\hline Distilled water upto $(\mathrm{ml})$ & 100 & 100 & 100 & 100 & 100 & 100 & 100 \\
\hline
\end{tabular}

Table 1: Preparation of Meloxicam In-situ gel formulation systems

\begin{tabular}{|c|c|c|c|c|c|c|c|c|c|c|c|c|c|c|c|c|c|}
\hline Ingredient & F8 & F9 & F10 & F11 & F12 & F13 & F14 & F15 & F16 & F17 & F18 & F19 & F20 & F21 & F22 & F23 & F24 \\
\hline Meloxicam (g) & 0.320 & 0.320 & 0.320 & 0.320 & 0.320 & 0.320 & 0.320 & 0.320 & 0.320 & 0.320 & 0.320 & 0.320 & 0.320 & 0.320 & 0.320 & 0.320 & 0.320 \\
\hline Sodium alginate (g) & 3 & 3 & 3 & 3 & 3 & 3 & 3 & 2 & 2 & 2 & 2 & - & - & - & - & - & - \\
\hline Xanthan gum (g) & 0.1 & - & - & - & - & - & - & - & - & - & - & 0.5 & 1 & - & - & - & - \\
\hline Gellan gum (g) & - & 0.1 & - & - & - & - & - & - & - & - & - & - & - & 0.5 & 1 & - & - \\
\hline Pectin (g) & - & - & 0.1 & - & - & - & - & - & - & - & - & - & - & - & - & 0.5 & 1 \\
\hline Chitosan (g) & - & - & - & 0.1 & - & - & - & 0.1 & - & - & - & - & - & - & - & - & - \\
\hline Guar gum (g) & - & - & - & - & 0.1 & - & - & - & 0.1 & - & - & - & - & - & - & - & - \\
\hline HPMC K4M (g) & - & - & - & - & - & 0.1 & - & - & - & 0.1 & - & - & - & - & - & - & - \\
\hline HPMC K15M (g) & - & - & - & - & - & - & 0.1 & - & - & - & 0.1 & - & - & - & - & - & - \\
\hline Sodium citrate (g) & 0.05 & 0.05 & 0.05 & 0.05 & 0.05 & 0.05 & 0.05 & 0.05 & 0.05 & 0.05 & 0.05 & 0.05 & 0.05 & 0.05 & 0.05 & 0.05 & 0.05 \\
\hline Calcium chloride (g) & 0.05 & 0.05 & 0.05 & 0.05 & 0.05 & 0.05 & 0.05 & 0.05 & 0.05 & 0.05 & 0.05 & 0.05 & 0.05 & 0.05 & 0.05 & 0.05 & 0.05 \\
\hline Distilled water & 100 & 100 & 100 & 100 & 100 & 100 & 100 & 100 & 100 & 100 & 100 & 100 & 100 & 100 & 100 & 100 & 100 \\
\hline
\end{tabular}

Formulation containing, xanthan gum(F8), Gellan gum (F9) and pectin(F10) have immediately formed gel without showing sol to gel conversions are not acceptable formulations. The F11- F14 formulations formed thick opaque gel upon transformation. Hence F15 (SA 2\%, Chitosan 0.1\%) F16 (SA 2\% guargum 0.1\%), F17 (SA2\% 
HPMC k4), F18 (SA 2\%, HPMC K15) having declining concentration of Sodium alginate (2\%) formed clear transparent gels upon transformation.

As F8, F9, F10 formulations were not shown sol to gel transformation instead directly formed gels, F19 to 24 formulations were prepared by polymers alone at concentrations of $0.5 \%$ and $1 \%$ without Sodium alginate incorporation.

Among F19 to 24, the formulations containing Xanthan gum alone (F19 \&F22) showed sol to gel change upon experience to $0.1 \mathrm{~N} \mathrm{HCl}$ but could'nt maintain the gel integrity for more than two hours of disintegration. The F23 \&F24 formulations containing Pectin alone formed direct gel instead of solution formulation so not accepted. Therefore further studies hence dissolution studies were planned for F11 to F18 and marketed SR Tablet.

\section{Fourier transform infrared (FTIR) spectroscopy}

The compatibility and interaction between drug and excipients utilized in the preparation of in situ gels are assessed by using FTIR-spectrophotometer $8400 \mathrm{~S}^{11}$. IR spectra's of lornaxicum and along with excepients are determined and scanning at range of 500 to $4000 \mathrm{Cm}^{-1}$ results are given in the Fig no. 2 .

\section{Differential scanning calorimeter (DSC) studies}

The samples thermal behavior was investigated by using DSC Q100. Accurately weighed required number of samples were taken in an aluminum pans then precisely crimped. At the rate of $20^{\circ} \mathrm{C} / \mathrm{min}$ from $-40^{\circ} \mathrm{C}$ to $300^{\circ} \mathrm{C}$ test samples were heated under stable nitrogen cleansing at a rate of $40 \mathrm{ml} / \mathrm{min}$.

\section{Evaluation of Meloxicam in situ gel}

The general appearance, color and odor of formulation were physically visualized and recorded and the $\mathrm{pH}$ was determined by using digital $\mathrm{pH}$ meter ${ }^{12}$.

\section{In-vitro gelation studies}

The formulations are subjected to know their in-vitro gelling capacity; accurately $10 \mathrm{ml}$ of measured each formulation was taken in a beaker and added $0.1 \mathrm{~N} \mathrm{HCl}$ $100 \mathrm{ml}, \mathrm{pH}$ of 1.2 followed by gentle agitation. The formed gels were observed and gel formed patterns are recorded.

\section{In vitro floating time}

The in-vitro floating studies were performed by introducing about $10 \mathrm{ml}$ of in-situ gel preparation was added to $100 \mathrm{ml}$ of $0.1 \mathrm{~N} \mathrm{HCl}, \mathrm{pH}$ of 1.2 at $37^{\circ} \mathrm{C}$. The time taken by the formulation to float was recorded.

\section{Drug content estimation}

Gel equivalent to $10 \mathrm{mg}$ of drug is transferred into a $100 \mathrm{ml}$ volumetric flask and initially dissolved in $20 \mathrm{ml}$ of $50 \%$ Methanol. Finally, the volume is fabricated to $100 \mathrm{~mL}$ with $50 \%$ Methanol. $1 \mathrm{ml}$ of this supernatant is then transferred into $10 \mathrm{ml}$ measuring flask and makes volume to $10 \mathrm{ml}$. Drug concentration was determined by using UV spectrophotometer against blank solution at wavelength of $276 \mathrm{~nm}$.

\section{Viscosity studies}

The viscosity of Meloxicam in-situ gels was measured for solution form and to gel form at $37^{\circ} \mathrm{C}$ by viscometer Brookfield DV Pro-II, United States. The testing samples are equilibrated in water thermo-stated jacket for $10 \mathrm{~min}$; measured at $50 \mathrm{rpm}$ with spindle no.65.

\section{In vitro drug release studies}

The In vitro drug release study was carried in a triplicate using USP II [paddle apparatus method] dissolution apparatus. The medium for dissolution studies was $900 \mathrm{ml}$ of $0.1 \mathrm{~N}$ hydrochloric acid as dissolution at $37 \stackrel{\circ}{ } \mathrm{C}$. The rate of stirring was $50 \mathrm{rpm}$. The maintained speed to be simulates in-vivo existing gentle agitation moreover be sluggish enough to avoid the infringement of gel formulation. At programmed time intermissions, accurately $5 \mathrm{ml}$ of sample was withdrawn and equivalent amount of fresh medium replaced at $37^{\circ} \mathrm{C}$ and the absorbance of the samples were measured at $373 \mathrm{~nm}$ using UV-Visible Spectrophotometer.

\section{Pharmacokinetic studies}

Twelve rabbits weighing $2-2.5 \mathrm{Kg}$, randomly divided into four groups, be used in this study as follows:

Group -1 - Control group, Group - 2 - Active Pharmaceutical Ingredient (Meloxicam), Group - 3 - Marketed formulation, Group - 4 - Optimized formulation.

Measurement of pharmacokinetic parameters of the treatments confirmed to guide lines of Institutional Animal Ethical committee having ID No. SVCP/IAE/2018. Each group consists of three rabbits. Each formulation was administered orally to the rabbits by oral feeding tube, and dose was followed considering the literature review. Animals were initially segregated based on their body mass and were divided into 4 groups of 3 each (groups were named as Blank, Marketed formulation, Pure drug and optimized formulation. Animals were maintained under standard laboratory conditions at $24 \pm 2^{\circ} \mathrm{C}$, relative humidity $50 \pm 15 \%$ and maintained under normal photoperiod ( $12 \mathrm{~h}$ dark/ $12 \mathrm{~h}$ light cycles) throughout the experiment. Blood samples were collected in $\mathrm{K}_{2}$ EDTA coated blood collection tubes from marginal ear vein as per the standard protocol. The sampling time intervals were $0.0,1.0,2.0,4.0,6.0,8.0,12.0$ and $24 \mathrm{hrs}$ respectively for each animal of all the groups. The blood samples were collected centrifuged at $4000 \mathrm{rpm}$ and the plasma was separated. Plasma samples were stored at $-20^{\circ} \mathrm{C}$ until analysis. Samples are examined for medicine concentrations by HPLC and PK parameters were studied. The PK parameters such as $t_{1 / 2}, T_{\max }, C_{\max }, A \cup C_{0-\infty}, A \cup C_{12}$ are estimated for all the formulations and compared to that of API. 


\section{X-ray imaging studies for floating of the formulation}

A protocol was designed for the x-ray studies to know floating time period of optimized In-situ gel formulation of Meloxicam. After getting authorization from Institutional Animal Ethical Committee, the studies were performed using White Newzeland Rabbits model. As per protocol the healthy rabbit weighing $2.5 \mathrm{~kg}$ which has housed for a minimum of 72 hours early to the study and had free access to water and food. Animals were kept for overnight fasting and prepared optimized Meloxicam In situ gel formulation along with radio opaque agent $\mathrm{BaCl}_{2}$ at the concentration of $15 \% \mathrm{w} / \mathrm{v}$ to ensure visibility by X-ray was administered orally. Throughout the experiment animal was not allowable to eat, but have free access to water. X- ray imaging studies were performed at programmed time intervals of $0,1,2,4,6,8$ and 12 hrs.

\section{RESULTS AND DISCUSSION}

\section{$\lambda_{\max }$ of Meloxicam}

The $\lambda_{\max }$ of Meloxicam: UV spectrum of Meloxicam showed maximum absorbance at $236 \mathrm{~nm}, 247 \mathrm{~nm}, 256 \mathrm{~nm}$ and $375 \mathrm{~nm}$ wave-length shown in figure no.1\&2. The calibration curve of Meloxicam by plotting concentration against absorbance results in a straight line and Better peak response and less placebo interference were observed at $375 \mathrm{~nm}$. Therefore wavelength of $375 \mathrm{~nm}$ is preferred to estimate the drug.

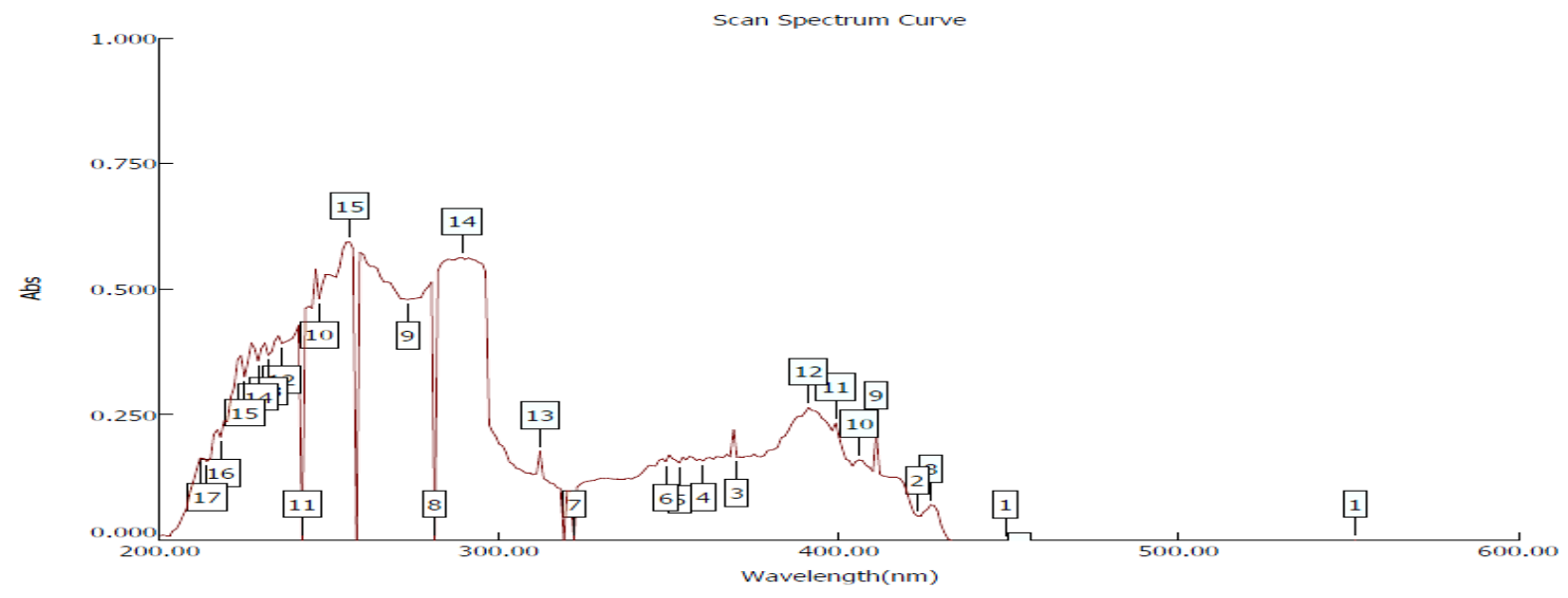

Figure 1: Meloxicam Absorption curve $\left(\lambda_{\max }: 375 \mathrm{~nm}\right)$

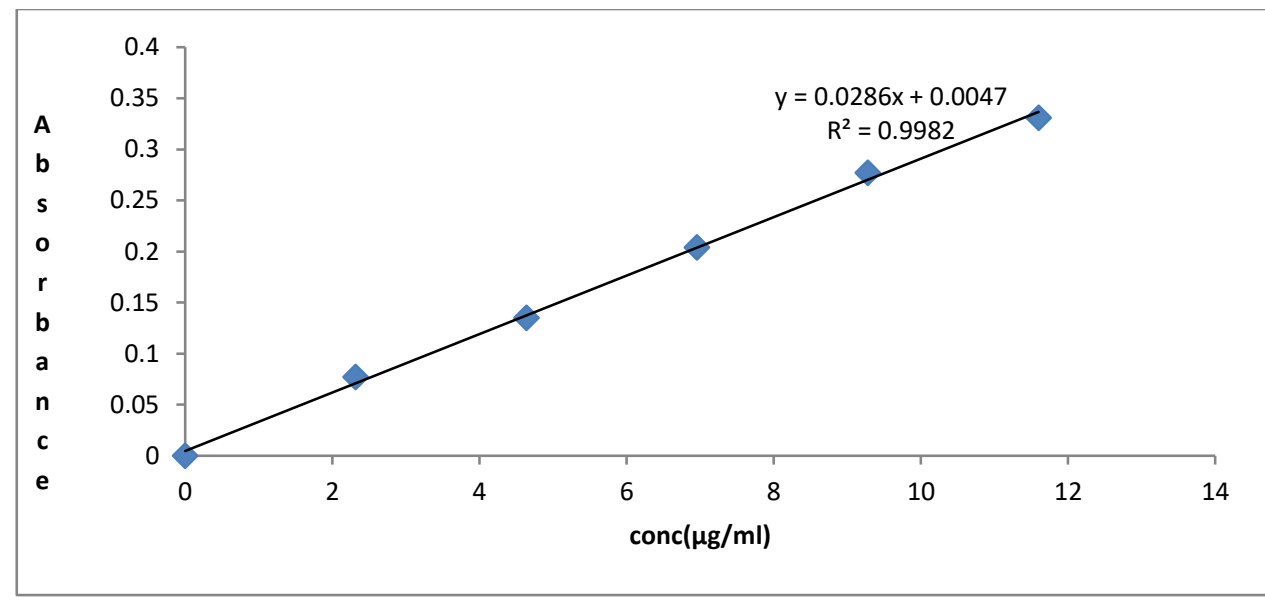

Figure 2: Calibration curve of Meloxicam

\section{FTIR and DSC studies}

The IR spectrum of Meloxicam pure, excipients of optimized formulation and Meloxicam optimized formulation illustrated that peaks characteristics of Meloxicam are not altered and with no changes in their spot, by this means representing no chemical drug interaction among drug and excipients. An IR spectral studies: confirmed that compatibility of Meloxicam with polymers utilized for in-situ gel preparations. The major peaks are obtained almost at the same wave numbers which belonging to drug functional groups. On the other hand, in the physical mixtures additional peaks were obtained due to attendance of impurities however there is no influence in the drug peaks. The observation of spectral studies has indicating that there was no significant change in the peaks of drug polymer mixture. Therefore, no specific interaction among drug and polymers was observed, outcomes of IR studies were given in fig no.3. The DSC studies of Meloxicam indicate that the obtained sample was a hydrate form result was shown in fig no.3. 

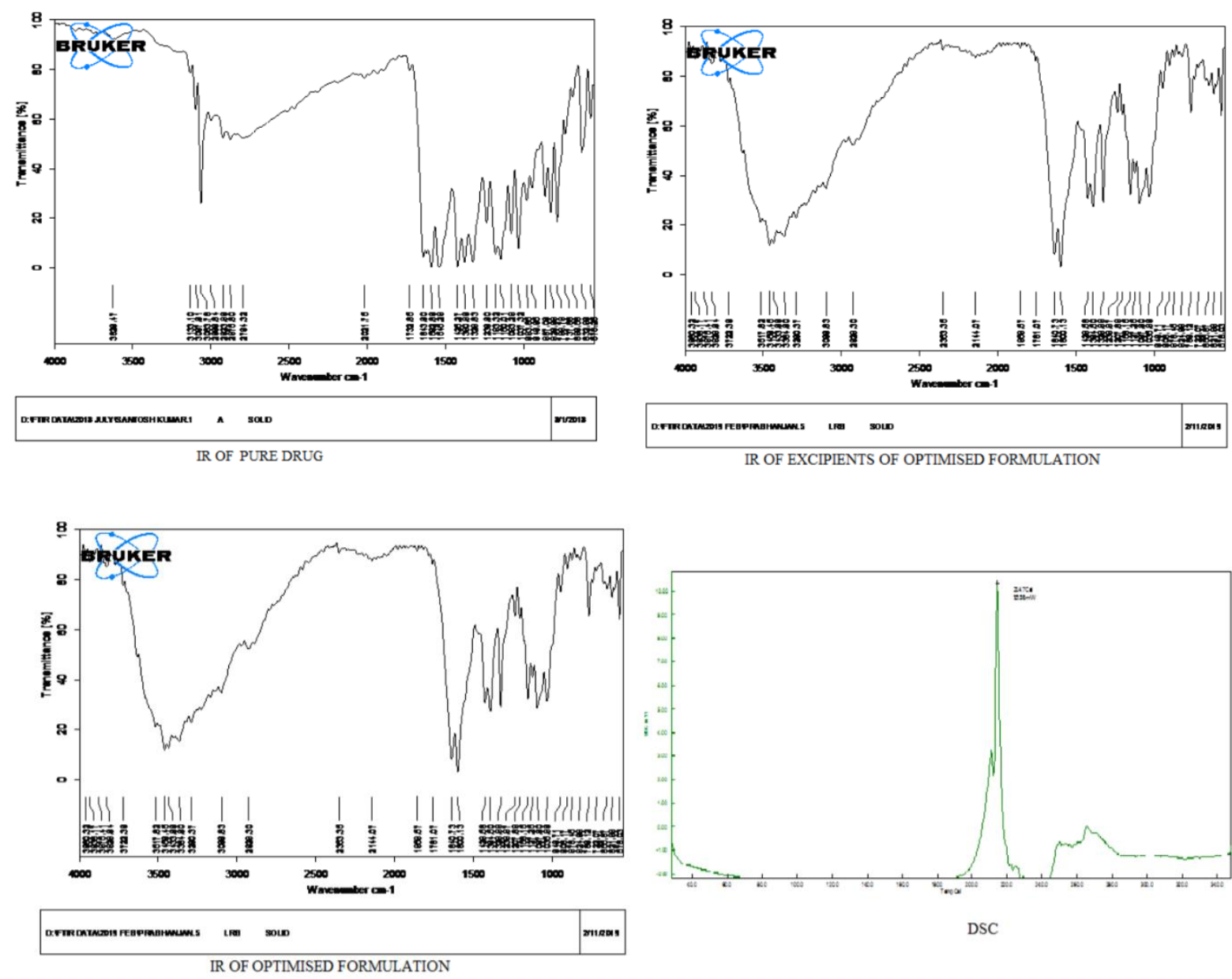

Figure 3: The IR spectra's of the Meloxicam, excipients of optimized formulation and Meloxicam optimized formulation and DSC of the Meloxicam.

\section{Physical appearance, $\mathrm{pH}$ and response of gelation}

The $\mathrm{pH}$ measurement is very essential for oral preparations; or else, it leads to annoyance to the gullet. All prepared formulations have to some extent acidic $\mathrm{pH}$. The formulations $\mathrm{pH}$ was founded in the range of 4.75-4.97 and physical appearance of formulations as shown in table no.2. Increasing concentration of Sodium Alginate resulted in producing an intact gel which could reside for longer period of time at concentration of $3 \%$ Sodium alginate and there is no much change in the gelling time and gel consistency by increasing concentration of calcium.

Table 2: Evaluation parameters (Gel appearance, $\mathrm{pH}$, Gelation response and \%Drug content) of in-situ gel formulations of Meloxicam

\begin{tabular}{|c|c|c|c|c|}
\hline Formulations & Gel appearance & $\mathbf{p H}$ & Gelation response & \%Drug content \\
\hline F11(C) & Opaque & 4.85 & +++ & $99.99 \%$ \\
\hline F12(G) & Opaque & 4.97 & +++ & $100.01 \%$ \\
\hline F13(Hk4) & Opaque & 4.77 & +++ & $98.99 \%$ \\
\hline F14(HK15) & Opaque & 4.95 & +++ & $99.01 \%$ \\
\hline F15 (CS2\%) & slightly opaque & 4.83 & +++ & $99.6 \%$ \\
\hline F16(GS2\% & slightly opaque & 4.93 & +++ & $101.04 \%$ \\
\hline F17 (HK4 S2\%) & slightly opaque & 4.75 & $\mathbf{+ + +}$ & $99.1 \%$ \\
\hline F18 (HK15 SA2\%) & slightly opaque & 4.92 & +++ & $99.5 \%$ \\
\hline
\end{tabular}

$(+++)$ : Immediate gelations remain for an extended period

\section{Determination of drug content}

Content of drug in all F11toF18 preparations were assessed and outcomes are in the acceptable range. The ranges of drug content values of formulations are in between 90 to $100 \%$ and the results were shown in the table no.2. 


\section{Viscosity and Floating studies}

The viscosities of the all-prepared formulations were low, a considerable increase was reported at due to conversion of sol-gel. The Meloxicam in-situ gel (F11) viscosity was 1321cps, a prominent raise was observed after geletion was $42068 \mathrm{cps}$. Taking into account all the results and findings of characterization, formulation F11 was considered for further studies results were shown in the table no.3.
Floating time and lag time were shown in table no. 3. As the concentration of crosslinking polymer resulted in change in viscosity, hence time taken from the sol to cohesive gelation and to appear on the surface of the medium was affected although signinficant difference is not observed. The in vitro floating test revealed the ability of all formulations to keep buoyancy for above $12 \mathrm{hr}$. All formulations exhibited total floating time of $>12 \mathrm{~h}$. Floating lag-time varied with formulation variables. Floating lag times of F11-F18 are in between 2-4sec.

Table 3: Evaluation parameters (Viscosity, floating lag time in sec and floating duration in hrs) of in-situ gel formulations of Meloxicam.

\begin{tabular}{|c|c|c|c|c|}
\hline \multirow{2}{*}{ Formulation } & \multicolumn{2}{|c|}{ Viscosity:(cps) } & $\begin{array}{c}\text { Floating : lag time } \\
\text { (sec) }\end{array}$ & $\begin{array}{c}\text { floating } \\
\text { duration (hrs) }\end{array}$ \\
\hline F11(C) & Formulation & Gel & 3 & $>12$ \\
\hline F12(G) & $1321 \pm 50$ & $42068 \pm 25$ & 4 & $>12$ \\
\hline F13(Hk4) & $1283 \pm 38$ & $41870 \pm 18$ & 3 & $>12$ \\
\hline F14(HK15) & $953 \pm 23$ & $31010 \pm 13$ & 4 & $>12$ \\
\hline F15(CS2\%) & $534 \pm 16$ & $25137 \pm 14$ & 2 & $>12$ \\
\hline F16(GS2\% & $572 \pm 16$ & $27245 \pm 22$ & 3 & $>12$ \\
\hline F17(HK4 S2\%) & $710 \pm 26$ & $26503 \pm 26$ & 2 & $>12$ \\
\hline F18(HK15 SA2\%) & $904 \pm 36$ & $31512 \pm 15$ & 3 & $>12$ \\
\hline
\end{tabular}

\section{In vitro drug release studies}

The In vitro drug release study results were graphycally shown in Fig.no.4. F11, F12, F13 and F14 released 96.12\%, $98.96 \%, 92.27 \%$ and $99.52 \%$ in $12,8,6$ and 8 hrs of dissolution. Formulations F15 and F16 released $98.32 \%$, 99.98\% in $6 \mathrm{hrs}$ and F17, F18 formulations released $99.78 \%$ and $96.71 \%$ in $4 \mathrm{hrs}$ of dissolution respectively.
Commercially available product SR, Meloxicam has showed $99.92 \%$ drug release in $8 \mathrm{hrs}$. Compare to all formulations based on the results of diffusion studies, F11can be considered as optimized formulation as aim of study is sustained release of the drug. Hence formulation F11 showing in vitro drug release of $99.52 \%$ over a $12 \mathrm{hrs}$ extended period.

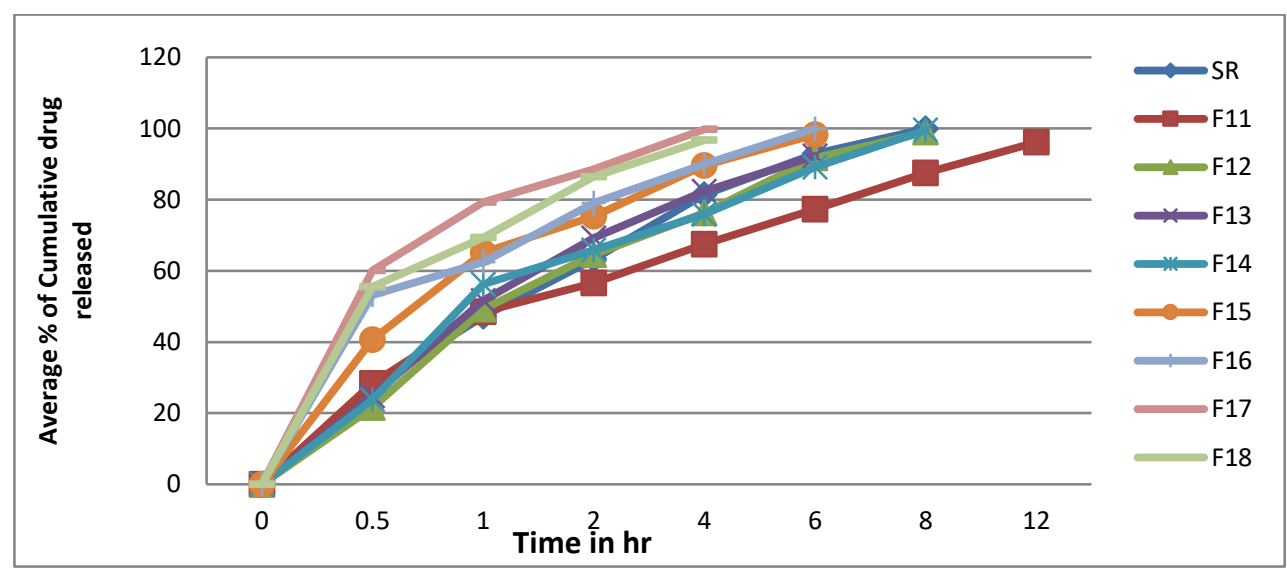

Figure 4: Comparative Summarization of in vitro dissolution studies of SR and F11 to F18

\section{Pharmacokinetic studies}

The percent of drug concentration in plasma was evaluated and plasma concentrations versus time curve for Meloxicam was shown in Fig.no.5. Pharmacokinetic parameters are presented in Table.no. 5. As compared to pure drug $(168.3 \mathrm{ng} / \mathrm{ml})$ F11 formulation showing Cmax value of $1121 \mathrm{ng} / \mathrm{ml}$ in blood and bioavailability and $\mathrm{T}_{\max }$ of F11 was $10 \mathrm{hrs}$, and pure drug was $8 \mathrm{hrs}$. AUC is an important parameter for evaluating bioavailability of drug from dosage form as it represents the total integrated area under the blood concentration time profile and represents the total amount of drug reaching. The F11 formulation and pure drug should have maximum variations in the case of AUC parameter. 
Table 4: Pharmacokinetic studies of optimised formulation F11, pure drug and marketed formulation.

\begin{tabular}{|c|c|c|c|c|c|c|}
\hline \multicolumn{7}{|c|}{ Pharmacokinetic Parameters for Meloxicam in Rabbits } \\
\hline Formulation & AUC $_{\mathbf{0} \rightarrow \mathbf{t}}$ & AUC $_{\mathbf{0} \rightarrow \infty}$ & $\mathbf{t}_{\mathbf{1} / \mathbf{2}}(\mathbf{h r s})$ & $\mathbf{C}_{\max }(\mathbf{n g} / \mathbf{m l})$ & $\mathbf{T}_{\max }(\mathbf{h r s})$ \\
\hline Pure Drug & 2767.15 & 389.563 & 6.69043 & 168.3 & 8 \\
\hline Marketed Formulation & 3660.2 & 502.565 & 7.16482 & 265.74 & 8 \\
\hline F11 & 14524.2 & 2735.82 & 9.09548 & 1121.05 & 10 \\
\hline
\end{tabular}

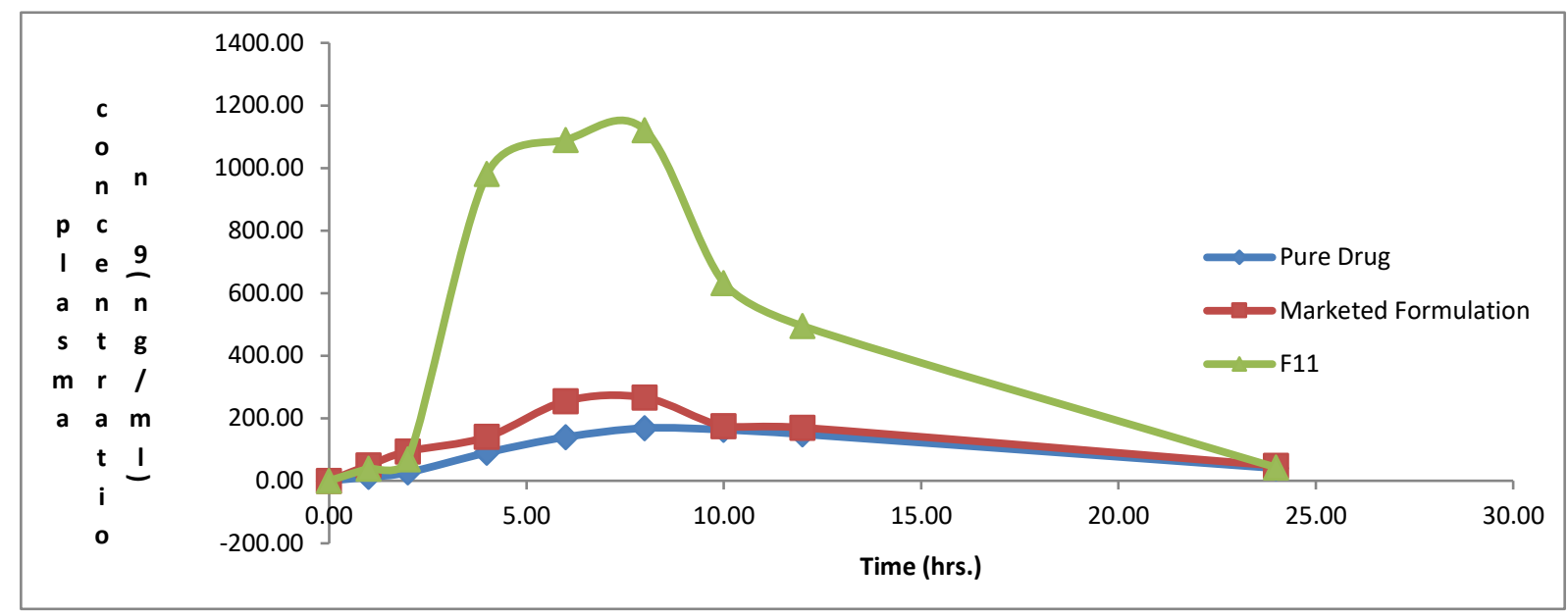

Figure 5: plasma concentrations verses time curve of formulations [F11], pure drug and marketed Formulation.

\section{In-vivo x-ray imaging studies}

An in-vivo Xray imaging study was performed in healthy Newzealand rabbit. The optimized formulation added with Barium Chloride at the concentration of $15 \% \mathrm{w} / \mathrm{v}$ was administered to rabbit through oral route and its abdomen X-ray images was taken to confirm the floating ability of optimized gel formulation (F11) of the Meloxicam. The Xray imagings were taken as i) X-ray image of Immediately
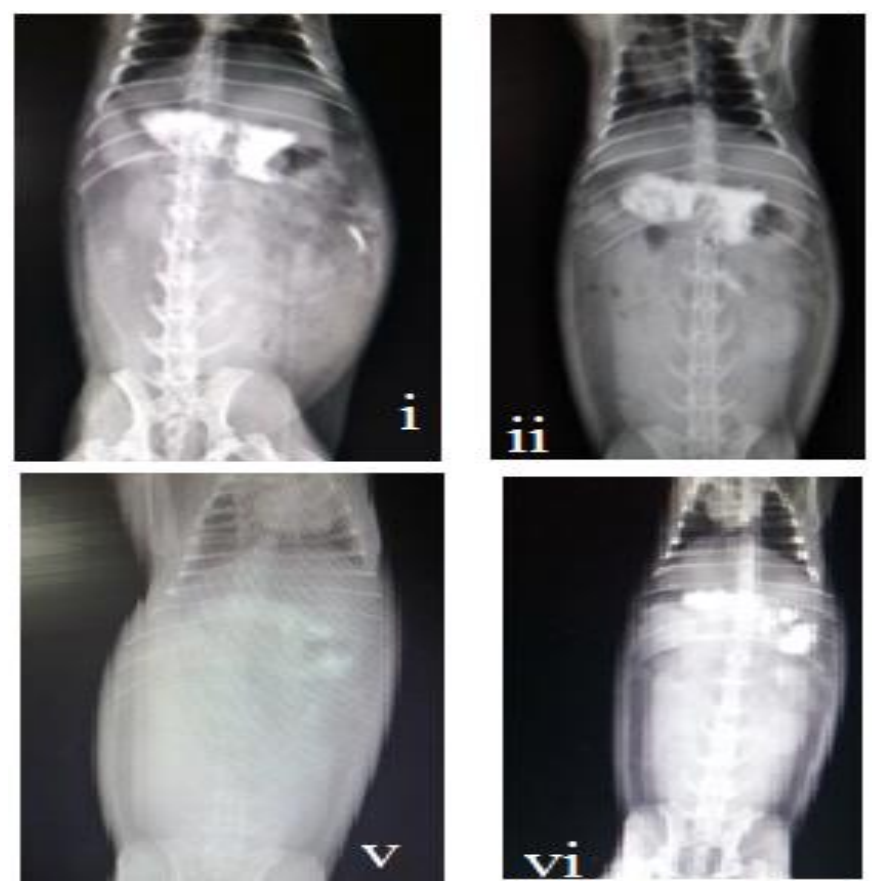

after administration ii) $X$ ray image After $1 \mathrm{hr}$ after gel administration iii) $X$-ray image after $2 \mathrm{hr}$. of gel administration and iv, v, vi, vii \& viii are $X$-ray images after $4,6,8,10$ and $12 \mathrm{hr}$ of gel administration. It was found that oral floating in-situ gel was float immediately after feeding to rabbit and it was observed to be floating in the stomach more than $12 \mathrm{hr}$ and results of $\mathrm{x}$-ray imaging's are given in fig no.6.
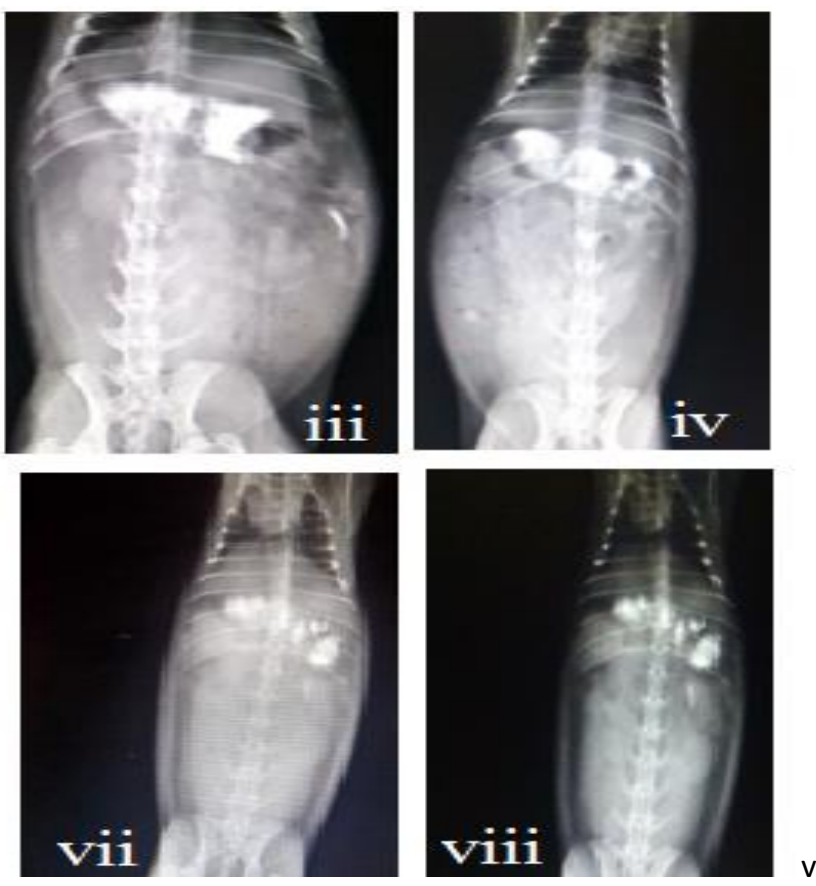

i) $\mathrm{x}$-ray image of Immediately after administration ii) x ray image After $1 \mathrm{hr}$ after gel administration iii) $\mathrm{X}$-ray image after $2 \mathrm{hr}$. of gel administration and iv, v, vi, vii \& viii are x-ray images after 4,6,8,10 and12 hr of gel administration.

Figure 6: In-vivo x-ray imaging studies of the Meloxicam optimized formulation. 


\section{CONCLUSION}

Commercial Meloxicam has short biological half-life and is rapidly eliminated, frequent oral administration is necessary to maintain its therapeutic concentration, the optimized formulation F11 having 3\% concentration of sodium alginate along with chitosan and calcium chloride had sustained effect improved than the marketed formulation basing on in-vitro drug release over an extended period of above $12 \mathrm{hrs}$. The X- ray imaging studies are confirming the sustainity in gel integrity maintenance which facilitates the continuous, sustained release of the drug instead of burst release. The in-vivo kinetic studies are approving the better performance of the optimized formulation in comparison to marketed formulation, the Cmax, Tmax, halflife AUC values are confirming the same thing. Hence, the in-situ gel formulation of Meloxicam containing sodium alginate along with chitosan as drug release controlling polymer as promising approach for the treatment of rheumatoid arthritis in a convenient dosage form with much patient compliance and better therapeutic response.

Acknowledgments: We are very grateful to Er. Ravi Chaudhary Founder \& Chancellor of K.K. University, Er. Richee Ravi Pro Chancellor of K.K. University and Dr. C. Venkataramana Reddy Vice Chancellor of K.K. University for patronage, encouragement, guidance, and financial support extended by them during the course of the study.

\section{REFEENCE}

1. Chopra, A., 2000. Ayurvedic medicine and arthritis. Rheum. Dis. Clin. North Am. 26; 133-144.

2. Lee, S.J., Kavanaugh, A., Pharmacological treatment of established rheumatoid arthritis. Best. Pract. Res. Clin. Rheumatol. 2003; 17: 811-829.

3. Pai, D.R., Prakash, V.M., Kumar, T.P., Current developments in therapeutic drug targeting for the management of rheumatoid arthritis: an emerging paradigm. Crit. Rev. Ther. Drug. 2019; 36 (6).

4. Misra, R., Sharma, B., Gupta, R., Pandya, S., Agarwal, S., Agarwal, P., Grover, S., Sarma, P., Wangjam, K., Indian rheumatology association consensus statement on the management of adults with rheumatoid arthritis. Indian. J. Rheumatol. 2008; 3: S1-S16.

5. Pincus, T., Aggressive treatment of early rheumatoid arthritis to prevent joint damage. Bull. Rheum. Dis. 1998; 47: 2-7.

6. Feldmann, M., Brennan, F., Maini, R., Role of cytokines in rheumatoid arthritis. Annu. Rev. Immunol. 1996; 14: 397440.

7. Butoescu, N., Jordan, O., Doelker, E., Intra-articular drug delivery systems for the treatment of rheumatic diseases: A review of the factors influencing their performance. Eur. J. Pharm. Biopharm. 2009; 73: 205-218.

8. Pincus, T., Callahan, L.F., Sale, W.G., Brooks, A.L., Payne, L.E., Vaughn, W.K., Severe functional declines, work disability, and increased mortality in seventyfive rheumatoid arthritis patients studied over nine years. Arthritis. Rheum. 1984; 27: 864-872.

9. Byrav DS, Medhi B, Prakash A, Patyar S, Wadhwa S. Meloxicam: A newer NSAID. Int J Pharm Med Research 2009; 20: 27-31.

10. Sivasubramanian L, Lakshmi KS, Tintu T. Simultaneous spectrophotometric estimation of paracetamol and Meloxicam in tablet dosage form. Int J Pharm Pharm Sci. 2010; $2: 166-8$.

11. Mulagada S, Baratam SR. Design and evaluation of ondansetron fast disintegrating tablets using natural polymers and modified starches as super disintegrants for the enhancement of dissolution. J Young Pharm 2017; 9: 519-24.

12. Chaudhary B, Verma S. Preparation and evaluation of novel in situ gels containing acyclovir for the treatment of oral herpes simplex virus infections. The Scientific World J 2014; 280: 9-28.

Source of Support: The author(s) received no financial support for the research, authorship, and/or publication of this article.

Conflict of Interest: The author(s) declared no potential conflicts of interest with respect to the research, authorship, and/or publication of this article.

For any question relates to this article, please reach us at: editor@globalresearchonline.net

New manuscripts for publication can be submitted at: submit@globalresearchonline.net and submit_ijpsrr@rediffmail.com 